\title{
Additive value of magnetic resonance neurography in diagnosis of brachial plexopathy: a cross-section descriptive study
}

Sara Mohamed Mahmoud Mabrouk ${ }^{1}$, Hossam Abd El Hafiz Zaytoon², Ashraf Mohamed Farid ${ }^{3}$ and Rania Sobhy Abou Khadrah ${ }^{2^{*}}$ (D)

\begin{abstract}
Background: Management of brachial plexopathy requires proper localization of the site and nature of nerve injury. Nerve conduction studies and electrophysiological studies (ED) are crucial when diagnosing brachial neuropathy but these do not determine the actual site of the lesion. Conventional MRI has been used to evaluate the brachial plexus. Still, it carried the disadvantage of the inability to provide multi-planar images that depict the entire length of the neural plexus. It might be difficult to differentiate the brachial plexus nerves from adjacent vascular structures. Magnetic resonance neurography (MRN) is an innovative imaging technique for direct imaging of the spinal nerves. Our study aims to detect the additive role of MRN in the diagnosis of brachial plexopathy over ED. Forty cases of clinically suspected and proved by clinical examination and ED—traumatic $(N=30)$ and non-traumatic $(N=10)$-were included in our study. We compared MRN finding with results of clinical examination and ED.

Results: MRN findings showed that the root was involved in $80 \%$ of cases, trunks in $70 \%$ of cases affecting the middle trunk in $40 \%$ of cases, the middle and posterior cord in $25 \%$, lateral cord in $50 \%$, and terminal branches on $10 \%$ of cases. Ten percent of cases were normal according to MRN, and 90\% had abnormal findings in the form of preganglionic nerve root avulsion in 30\% of cases, mild perineural edema surrounding C6/7 nerve roots in 20\%, lower brachial trunk high signal in 10\%, complicated with pseudo meningocele in 20\%, and with increased shoulder muscle T2 signal intensity with muscle atrophy in 10\%. There were minimal differences between clinical examination finding and MRN findings, with very good agreement between electromyography and nerve conduction ( $p$ value $<0.05$, with sensitivity and specificity values of $94.44 \%$ and $100 \%$, respectively).
\end{abstract}

Conclusion: MRN is important in differentiating different types of nerve injuries, nerve root avulsion, and nerve edema, playing an important role in differentiating the site of nerve injury, both preganglionic or postganglionic and planning for treatment of the cause of nerve injury, either medical or surgical.

Keywords: Magnetic resonance neurography, Plexopathy, Electrodiagnostic, Brachial plexuses

\footnotetext{
* Correspondence: rotinia2009@gmail.com

${ }^{2}$ Department of Radiology, Faculty of Medicine, Tanta University Hospital,

Gharbia Governorate, Tanta, Algish Street, 31511, Egypt
}

Full list of author information is available at the end of the article

\section{Springer Open}

(c) The Author(s). 2021 Open Access This article is licensed under a Creative Commons Attribution 4.0 International License, which permits use, sharing, adaptation, distribution and reproduction in any medium or format, as long as you give appropriate credit to the original author(s) and the source, provide a link to the Creative Commons licence, and indicate if changes were made. The images or other third party material in this article are included in the article's Creative Commons licence, unless indicated otherwise in a credit line to the material. If material is not included in the article's Creative Commons licence and your intended use is not permitted by statutory regulation or exceeds the permitted use, you will need to obtain permission directly from the copyright holder. To view a copy of this licence, visit http://creativecommons.org/licenses/by/4.0/. 


\section{Background}

The brachial plexus is a network of nerves formed by the ventral branches of the spinal nerves C5-T1 in the posterior triangle of the neck. It is responsible for motor and sensory innervation to the upper extremity $[1,2]$. The roots of the brachial plexus are combined to form three trunks, which were divided to form anterior and posterior divisions. At the level of the lateral margin of the first rib, the divisions are combined to form three cords, which give rise to the peripheral nerves of the upper limb in the axilla [3].

Pathology of the brachial plexus varies widely; this is due to similar mechanisms of injury based on age. For example, in neonates, the most common pathology is related to birth trauma. In adolescents and young adults, pathology is often due to high-speed trauma, such as motor vehicle accidents. In adults, neoplasms (intrinsic or extrinsic masses) or post-radiation injury are frequently the cause. Traumatic injuries to the brachial plexus are associated with weakness and paresthesia of the upper extremity on the affected side. A thorough neurologic examination can be performed to localize the injury and to help pinpoint the location of pathology $[4,5]$.

Conventional magnetic resonance imaging MRI is applied to evaluate the brachial plexus. A combination of fatsuppressed T2-weighted either frequency-selective or short tau inversion recovery [STIR] sequences and T1-weighted MR sequences are used, and the major disadvantage of these sequences is their inability to provide multi-planar or projection images that cover the entire length of the neural plexus. It may be difficult to differentiate the brachial plexus nerves from adjacent vascular structures [6-8].

Magnetic resonance neurography (MRN) is an imaging technique that combines MRI with specially designed phased-array surface coils that allow for visualization of the peripheral nerves [9]. The surface coil technology is combined with highly effective fat-suppressed T2weighted MR pulse sequences to best visualize the nerves among other soft tissues. Compared to conventional MRI techniques, MRN features rapid image acquisition and high resolution capable of showing the small fascicular organization of nerves to detect different types of extraneural and intraneural lesions. It has been increasingly used in assessing lesions affecting peripheral nerves, plexus, and spinal nerve roots $[10,11]$.

We aim to detect the additive role of MRN in the diagnosis of brachial plexopathy over (ED) using correlation of MRI findings with the clinical and ED for better diagnosis of the cause and injury site to improve management of these cases.

\section{Methods}

Our retrospective and descriptive cross-section study was carried out on 40 patients who complained of motor weakness or sensory affection and were referred by neurologists, neurosurgeons, or oncologists to our radiodiagnosis department with clinical suspicion of a brachial plexus injury from October 2018 to December 2020. The ethical committee of our institution has approved this study. We were informed of written consent from participants. The inclusion criteria were patients diagnosed with brachial plexopathies either by clinical findings or positive ED findings, including postoperative cases; the exclusion criteria were patients who had a bilateral complaint, postoperative cases, patients who did not have ED study, had any artificial metallic processes, claustrophobia, and patients who had pain due to shoulder joint de-arrangement. All patients in this study were subjected to the following:

1. Detailed history-taking including the personal history, risk factors as hypertension, diabetes mellitus, obesity, drug intake, smoking, and comorbid diseases including renal, hepatic diseases, or collagen disorders. History of trauma, radiotherapy, or operation.

2. Clinical examination: neurological examination, motor, and a sensory evaluation by physician.

3. Investigational studies: electrophysiological studies (ED), including nerve conduction study and electromyography (EMG).

4. Radiological investigation: magnetic resonance neurography (MRN)

\section{MR image acquisition}

All patients were examined with a 1.5 Tesla MR system (GE Healthcare, New York, USA). Patients were imaged in the supine position with arms at their sides so that the longitudinal alignment light lies in the midline and the horizontal alignment light passes through the level of the sternoclavicular joints. A neurovascular (NV) phased array coil was used. The time of examination was about $30 \mathrm{~min}$. We advised patients to avoid swallowing as possible during examination. The head entered the scanner first.

\section{MR sequences}

Our MRN examination protocol for the evaluation of the brachial plexus (FOVs from C 2 to $\mathrm{T} 2$ ) was shown in Table 1. Sagittal plane imaging for demonstration of the mid- and distal portions of the brachial plexus, because the nerves are seen in cross-section and their exact relationship to the surrounding vascular and soft tissues structures can be detected. Coronal plane imaging for visualization of the supraclavicular portion of the brachial plexus allow assessment of side-to-side asymmetry. Axial plane imaging allows for assessment of side-to-side asymmetry for evaluation of the long axis of the proximal nerves of the plexus.

The following sequences were taken (Table 1): coronal T2 WI, coronal T1 WI, coronal 3D FIESTA, coronal 
Table 1 MRN examination protocol for the evaluation of the brachial plexus (FOVs from C2 to T2)

\begin{tabular}{lll}
\hline Sequences & TR & TE \\
\hline Coronal T2-weighted & 4279.0 & 103.4 \\
Coronal T1-weighted & 489.0 & 9.33 \\
Coronal 3D FIESTA(SSFP). & 6.32 & 34 \\
Coronal STIR & 3548 & 41.9 \\
Axial 2D FIESTA(SSFP) FATSAT & 5.7 & 2.66 \\
Axial T1 & 526 & 9.8 \\
Coronal 2D FIESTA (SSFP)FATSAT & 5.4 & 2.5 \\
Sagittal STIR & 6099 & 43.3 \\
Axial 3D FIESTA (SSFP). & 5.7 & 2.1 \\
\hline
\end{tabular}

${ }^{a} T F$ indicates turbo factor, SPAIR spectral-attenuated inversion recovery, STIR short tau inversion recovery, FIESTA (SSFP (steady-state free precession) sequence, known by its different acronyms such as FIESTA (GE)

STIR, an axial 2D FIESTA FATSAT, an axial T1, coronal 2D FIESTA FATSAT, sagittal STIR, and an axial 3D FIESTA. The most important sequence that showed the neural anatomical details was 3D FIASTA SSFP (steadystate free precession).

\section{Image processing and analysis}

Post-acquisition reconstruction of $3 \mathrm{D}$ sequences was done including MPR (multi-planar reconstruction) and MIP (maximum intensity projection). Two radiologists reviewed images of the diseased side with at least 5 years of experience in MRN. The brachial plexus in both sides were evaluated by examining the ganglia, roots, trunks, divisions, and cords giving up to 16 segments for each patient.

\section{Statistical analysis}

IBM SPSS statistics (V. 25.0, IBM Corp., USA, 2016) was used for data analysis. The diagnostic validity tests were done including the diagnostic sensitivity, the diagnostic specificity, a +ve predictive value, a -ve predictive value, and the diagnostic accuracy of the test. We used chisquare $(\chi 2)$ test, Fisher's exact (FE), and Kappa test (K) to assess a statistically significant difference, with $p$ value $<0.05$ considered significant. Mean values were presented \pm SDs. We expressed Kappa values in intervals: < 0.2 poor, 0.21-0.4 fair, 0.41-0.60 moderate, 0.61-0.80 good, 0.81-0.99 excellent, and 1 perfect.

\section{Results}

This descriptive study involved 40 (22 males (55\%) and 18 females (45\%)) cases of diagnosed brachial plexus injuries either clinically or by ED studies, and their age ranged from 7 to 60 years with mean age $28.5 \pm 16.4$ SD. Patients' complaints were pain in 20 patients $(50 \%)$, muscle weakness in 12 patients (60\%), and tingling in 6 cases (30\%). The right-sided lesions were 24 cases (60\%)

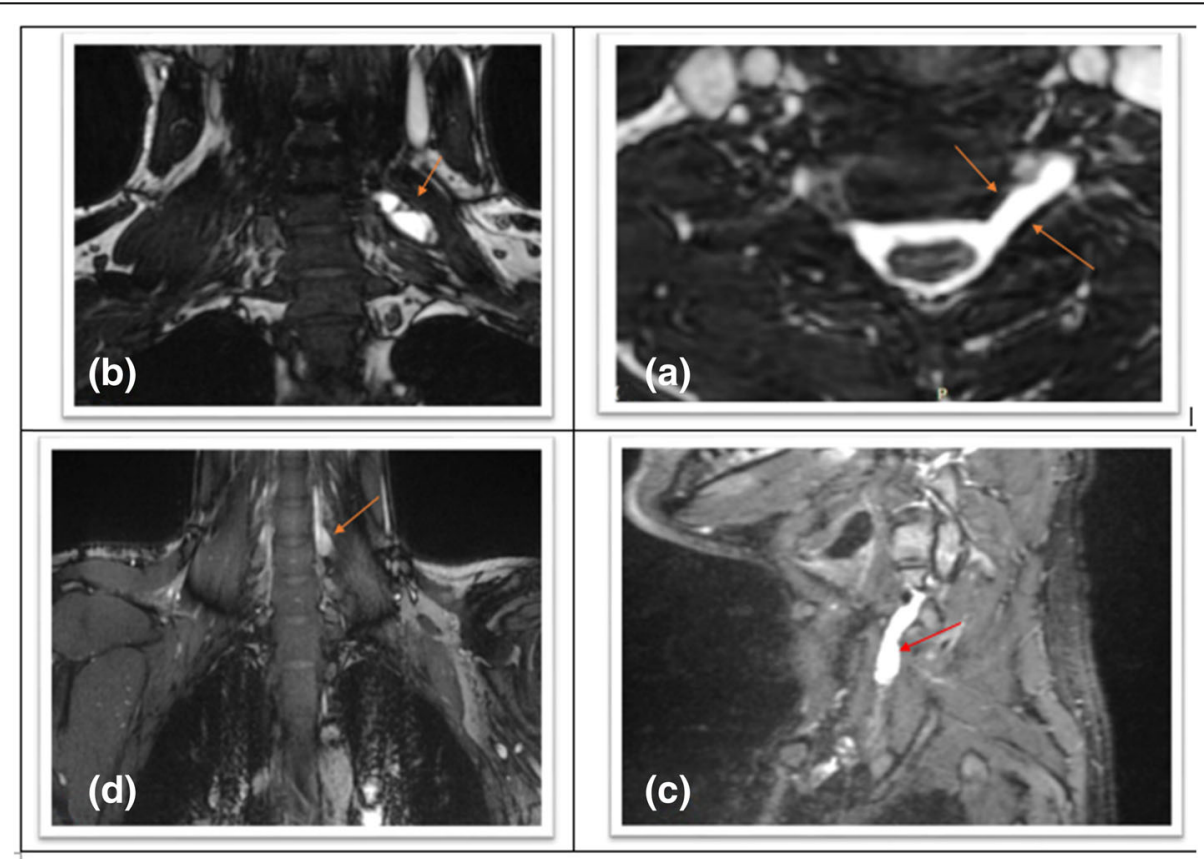

Fig. 1 A 23-year-old male patient represented by post-traumatic hypesthesia over C5 and C6 nerve distribution. The electrodiagnostic study showed injury at the level of roots (root avulsion), preganglionic lesion, and complete degeneration of C4, C5, and C6 nerves. a Axial 3D VIESTA image showed left preganglionic nerve root avulsion with pseudo meningocele formation. b Coronal 3D FIESTA. c Sagittal STIR images showed pseudo meningocele formation opposite to the left C5 and C6 nerve roots with perineural edema surrounding left C7 nerve root. $\mathbf{d}$ Coronal 3D FIESTA FAT SAT showed decrease in the muscle bulk on the left shoulder girdle with heterogeneous muscle SI denoting its denervation (arrow) 
while left-sided lesions were 16 (40\%). Time of patients MRI examination from the beginning of complaints was between 2 and 5 months with mean 3.77 months. The causes of brachial plexus injury were traumatic (24 cases) (Figs. 1, 2, 3, and 4) due to nerve trauma either congenital birth trauma or direct trauma (60\%), nontraumatic (16 cases) due to disc compression (12 cases $(30 \%))$, or neoplastic affection either primary or metastatic (8 cases $(20 \%))$. On the basis of clinical examination (sensory and reflexes), 24 patients were normal and 16 patients had diminished sensation, while reflexes were normal in 28 cases and decreased in 12 cases (Table 2). According to ED studies, 4 (10\%) cases were normal, 36 (90\%) were with abnormal findings either nerve conduction or motor affection as follows: 20 with abnormal nerve conduction, 4 with abnormal motor function, and 20 with both motor and nerve conduction abnormalities as shown in Table 3.

According to our MRN finding of brachial plexuses segment affection tabulated in Table 4, we noticed root involved in $80 \%$ of cases and trunks in $70 \%$ of cases and most of them were the middle trunk, cord affection was found in $40 \%$ of cases and involved middle, posterior cord in $25 \%$, lateral cord in $50 \%$, and terminal branches affection in $10 \%$ of cases. The most detected abnormalities in the muscles were muscles edema (36 cases $(90 \%))$, fatty infiltration (32 cases $(80 \%))$, and muscle atrophy (16 cases (40\%)).

MRN diagnosis of 40 studied cases of suspected brachial plexopathy was tabulated in Table 5. This table shows that $10 \%$ of cases (4 cases) were normal according to MRN and 90\% (36) had abnormal finding in the form of complete avulsion with pseudo meningocele and distal changes in the form of edema of distal trunks and divisions (24), distal muscle affection in 18 cases $(18 / 24)$, brachial neuritis in the form of nerve thickening and high SI in FIASTA sequences $20 \%$ (8 cases), brachial neuritis in the form of nerve thickening and high SI in FIASTA sequences with distal muscle affection $20 \%$ (8 cases) as muscle edema (high SI), decrease in the muscle bulk, and muscle atrophy or combination of them. There was a minimal difference between the clinical examination finding and MRN finding regarding involved roots, trunks, cord, and terminal branches.

When MRN findings were compared to the clinical examinations and electrophysiological studies as tabulated in Table 6, there was a good agreement between electromyography, nerve conduction and MRN, MRN sensitivity, and specificity were $94.44 \%$ and $100 \%$, respectively.

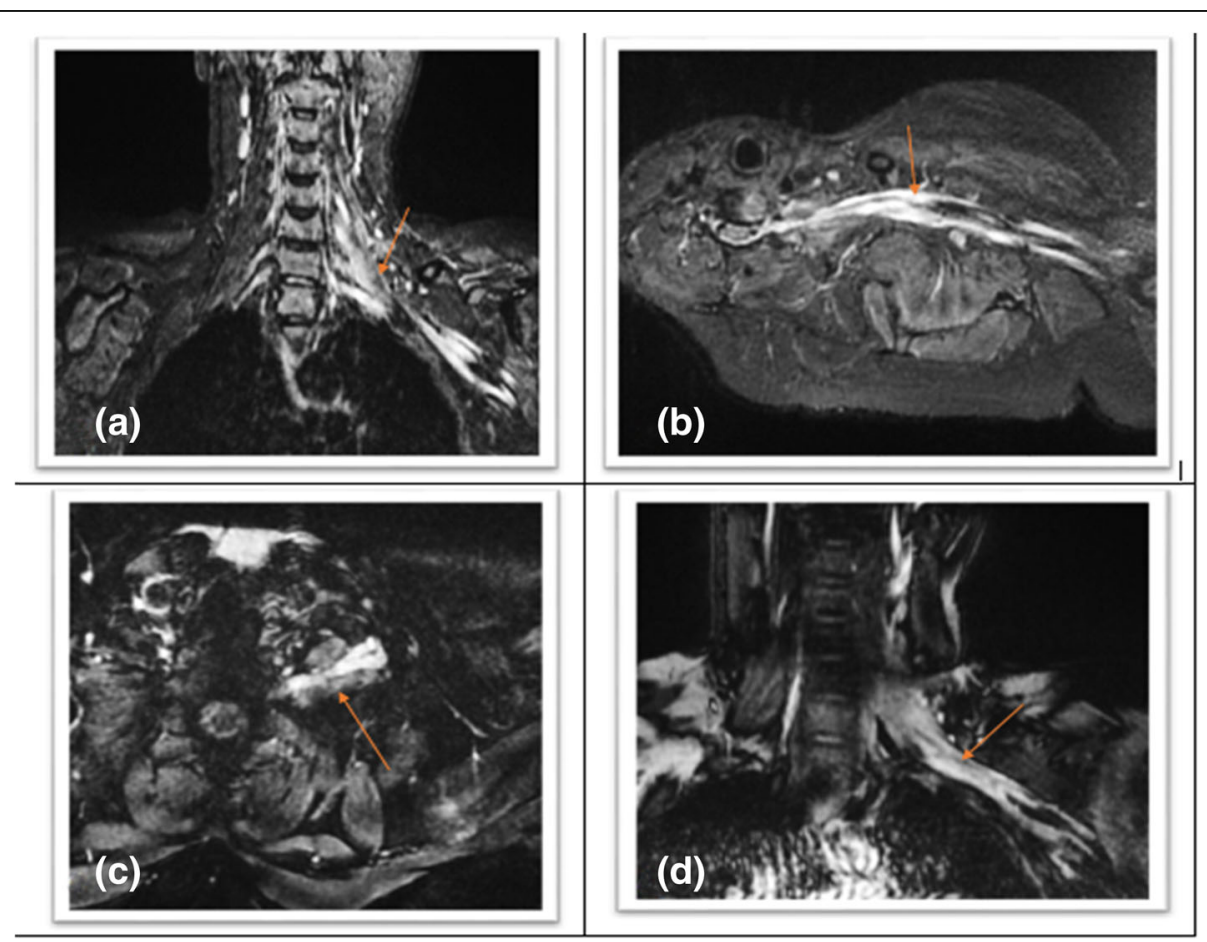

Fig. 2 A 37-year-old female patient complained of weakness of left upper limb with pain and tingling after sudden dislodgement of central cannula. Electrodiagnostic studies showed evidence of brachial plexus lesion in the form of complete subacute axonal lesion affecting upper, middle trunks (neurotemesis), and partial subacute axonal lesion affecting lower trunk (axonotemesis). MRN revealed a coronal STIR-weighted image and $\mathbf{b}$ sagittal reconstruction STIR-weighted image. $\mathbf{c}$, $\mathbf{d}$ Axial and coronal 3D FIESTA FAT SAT shows the left brachial plexus with increased thickness with mild perineural edema due to compression with ill-defined intermuscular edema related to the thoracic inlet (arrow) 


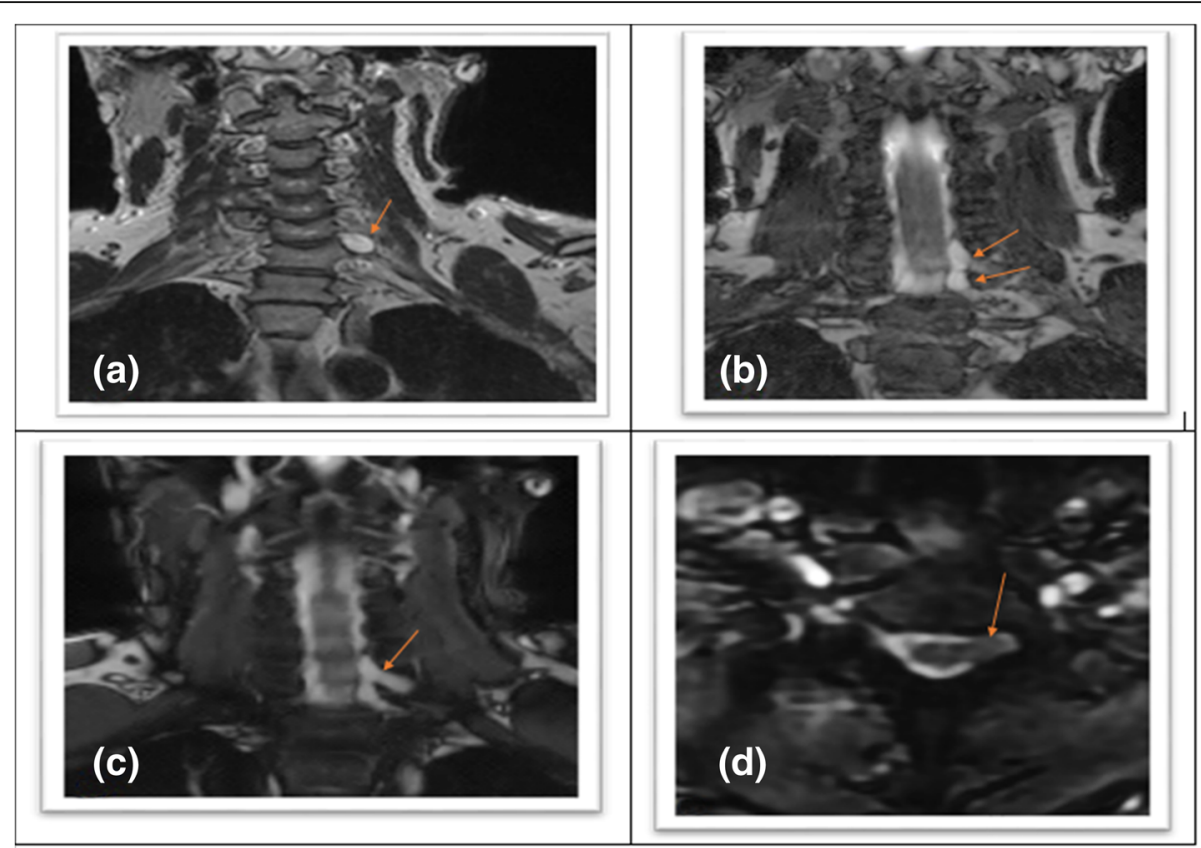

Fig. 3 A 15-year-old male patient had loss of function of the left upper extremity since birth Erb's paralysis, electrodiagnostic studies suggested post-traumatic injury at the level of roots (root avulsion) at C6 and C7. MRN revealed a coronal T2, b coronal 3D VIESTA, c coronal 2D FIESTA FAT SAT, and $\mathbf{d}$ axial 2D VIESTA FAT SAT obtained images show evidence of root avulsion at the level of left C6-7 root (arrow) with CSF sac seen in place likely traumatic meningocele (arrow)

\section{Discussion}

MRN is widely being accepted for nerve imaging, as it exhibits high diagnostic performance while inspecting brachial plexus injuries and helps in conducting further analysis of the cause, the site, and the nature of the injury [12]. In our study, we found minimal differences between the clinical examination findings and the MRN findings, and good agreement between electromyography, nerve conduction, and the MRN finding, with a sensitivity of $94.44 \%$ and a specificity of $100 \%$ (kappa 0.773, $\mathrm{P} 1 \frac{1}{4}$ 0.016). Another study by Fisher et al. [13] compared ED with MRN and showed that the ED tests
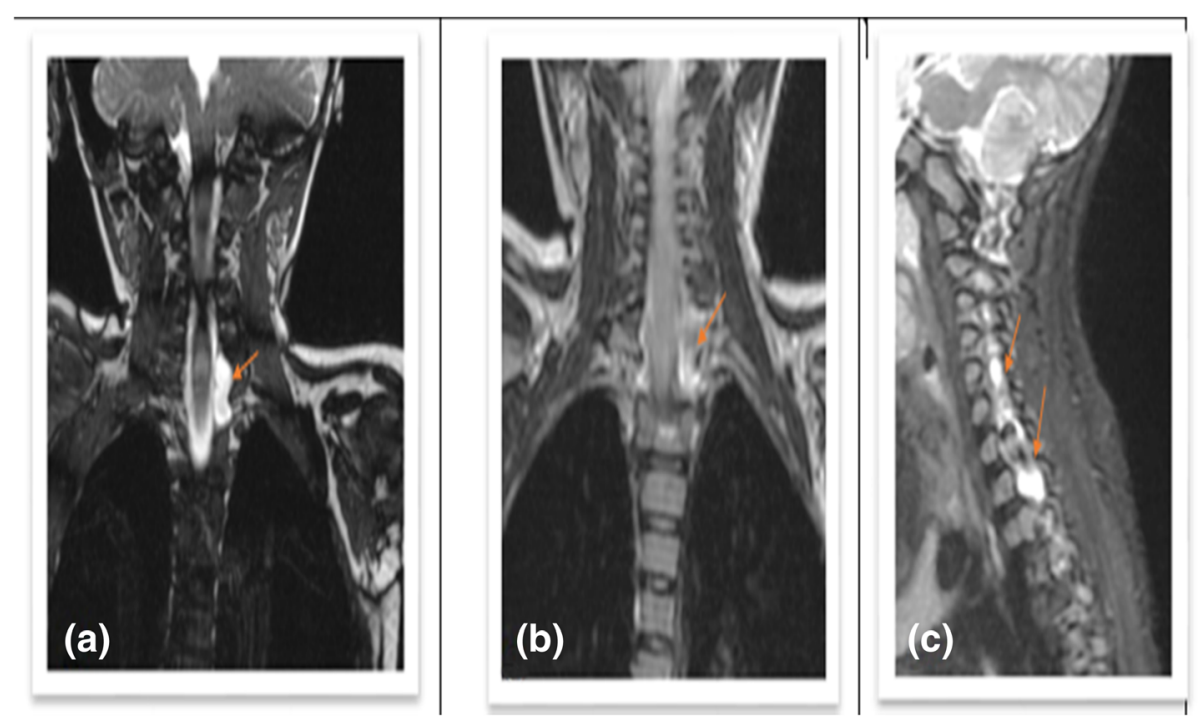

Fig. 4 A 3-year-old female child complained of left Erb's palsy: electrodiagnostic studies suggested left total complete root avulsion of all roots (C5, C6, C7, C8, and T1). a Coronal 3D FIESTA. c Sagittal STIR showed abnormal left well-defined lobulated lesion opposite to C7 and T1 (pseudo meningocele). $\mathbf{b}$ Coronal T2 showed the abnormal high signal at the left lower trunk of the brachial plexus 
Table 2 Distribution of the studied cases according to clinical findings

\begin{tabular}{lll}
\hline & No & $\%$ \\
\hline Sensory examination & & \\
Normal & 24 & 60.0 \\
Diminished & 16 & 40.0 \\
Reflexes & & \\
Normal & 28 & 70.0 \\
Diminished & 18 & 30.0 \\
\hline
\end{tabular}

were concordant with the MRN findings in 31 (66.0\%) of 47 cases. MRN imaging preceded the ED tests in 21 (44.7\%) of 47 cases. Another study by Smith et al. [14] showed that there was a greater correlation between the MRN and physical examination findings (kappa 0.6715, coefficient of correlation 0.7110, $P<0.001$ ) than between the EMG and the physical examination findings (kappa 0.5748, coefficient of correlation 0.5883, $\mathrm{P} 1 / 4$ 0.0012). Contrary to our results, Du et al. [15] showed that when MRN was compared with EMG/NCS, MRN was found to give the same information in 29 patients (32\%), additional diagnostic information in 41 (45\%), less in $15(17 \%)$, and a different diagnosis in 6 (7\%). They explained that MRN provided less diagnostic information when no abnormality was shown, or the area of abnormality was not included in the scan (for example, if the brachial plexus was scanned when the lesion was in the distal ulnar nerve). This difference in the findings and correlation between the ED studies and MRN may be attributed to their use of a 3 Tesla MRI machine, while our study used a 1.5 Tesla machine.

In our study, we found that the electrophysiological tests were negative in 4 cases $(10 \%)$ and positive in 36 (90\%) confirming the MRN diagnosis. Our MRN findings regarding the level of detected lesions involved as a proportion of cases were as follows: root $80 \%$, trunks in $70 \%$ (mainly the middle trunk), cord affection 40\%,

Table 3 Distribution of the studied cases according to electromyography and nerve conduction $(n=40)$

\begin{tabular}{lll}
\hline Electromyography and nerve conduction & No. & $\%$ \\
\hline Normal & 4 & 10.0 \\
Abnormal & 36 & 90.0 \\
At level of roots (root avulsion) & 4 & 10.0 \\
$\begin{array}{l}\text { Complete subacute axonal lesion affecting } \\
\text { upper, middle trunks, partial subacute axonal } \\
\text { lesion affecting lower trunk }\end{array}$ & 12 & 30.0 \\
$\begin{array}{l}\text { Total complete root avulsion of all roots } \\
\text { (C5, 6, 7, 8, T1) }\end{array}$ & 12 & 30.0 \\
Root avulsion (C6, 7) & & 10.0 \\
$\begin{array}{l}\text { Degeneration of C8-T1 roots, consequent } \\
\text { affection of median ulnar }\end{array}$ & 4 & 10.0 \\
\hline
\end{tabular}

Table 4 MRN diagnosis of studied cases $(n=40)$ of brachial plexopathy

\begin{tabular}{lll}
\hline MRN diagnosis & No. & $\%$ \\
\hline Normal & 4 & 10.0 \\
Abnormal & 36 & 90.0 \\
Mild perineural edema surrounding C6/7 nerve roots & 8 & 20.0 \\
Lower brachial trunk high signal & 4 & 10.0 \\
Preganglionic nerve root avulsion & 12 & 30.0 \\
Pseudo meningocele C5/6/7 & 8 & 20.0 \\
Increased shoulder muscle intensity with muscle atrophy & 4 & 10.0
\end{tabular}

involved middle and posterior cord in $25 \%$, lateral cord in $50 \%$ and terminal branches affection in $10 \%$ of cases. The MRN study of brachial plexuses by Upadhyaya et al., however, revealed the percentage of lesions per case: roots in $88 \%$, trunks in $80 \%$, and cords in $88 \%$. This implies a high incidence of injury in both the proximal and distal plexuses. Yoshida et al. [16] also showed that the nerve root affection was founded in most patients. The distribution of nerve root signal abnormalities and foraminal stenosis showed a significant correlation.

Our study has been claimed that the cause of brachial plexopathy was per number of cases: preganglionic nerve root avulsion (30\%), mild perineural edema surrounding C6/7 nerve roots $(20 \%)$, lower brachial trunk high signal (10\%), pseudo meningocele (20\%), and with increased shoulder muscle T2 signal intensity with muscle atrophy in $10 \%$ of cases.

Upadhyaya et al.s [17] MRN findings suggested root avulsion in 11 patients (44\%) with 31 avulsed roots. The percentage of avulsed roots at C5, C6, C7, C8, and T1 levels was $12.9,29.0,32.2,19.3$, and 6.4 , respectively. In

Table 5 Comparison between MRN and clinical examination finding at different levels of brachial plexuses injury

\begin{tabular}{|c|c|c|c|c|c|}
\hline & \multicolumn{2}{|c|}{ MRN } & \multicolumn{2}{|c|}{ Clinical examination finding } & \multirow[t]{2}{*}{$\overline{M_{P N}}$} \\
\hline & No. & $\%$ & No. & $\%$ & \\
\hline \multicolumn{6}{|c|}{ Roots involved } \\
\hline No & 8 & 20.0 & 16 & 40.0 & 0.125 \\
\hline Yes & 32 & 80.0 & 24 & 60.0 & \\
\hline \multicolumn{6}{|l|}{ Trunks } \\
\hline No & 12 & 30.0 & 16 & 40.0 & 0.500 \\
\hline Yes & 28 & 70.0 & 24 & 60.0 & \\
\hline \multicolumn{6}{|l|}{ Cords } \\
\hline No & 24 & 60.0 & 24 & 60.0 & 1.000 \\
\hline Yes & 16 & 40.0 & 16 & 40.0 & \\
\hline \multicolumn{6}{|c|}{ Terminal branches } \\
\hline No & 36 & 90.0 & 40 & 100.0 & 0.500 \\
\hline Yes & 4 & 10.0 & 0 & 0.0 & \\
\hline
\end{tabular}


Table 6 Agreement for electromyography and nerve conduction

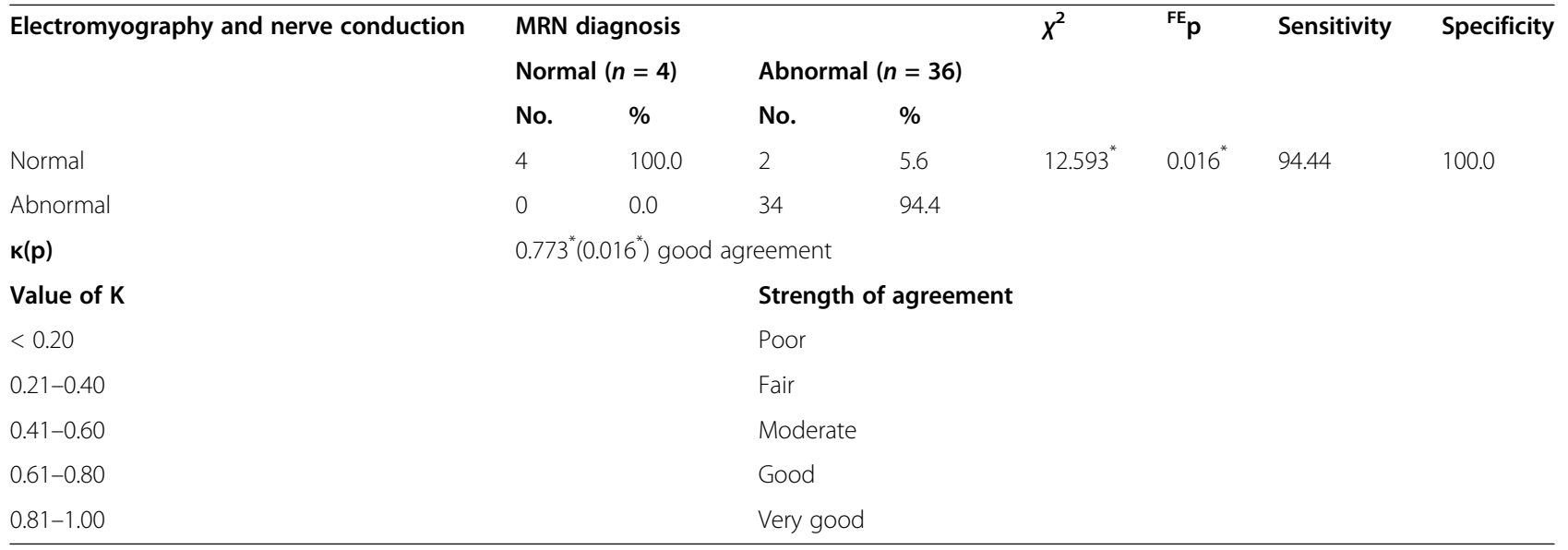

$X 2$ chi-square test, FE Fisher's exact, $k$ kappa test

*Statistically significant at $p \leq 0.05$

agreement with Gad et al. [12], Vargas et al. [1], and Somashekar et al. [18], most of pervious MRN studies used the traditional MR sequences (T1WI, T2WI, and STAIR). We used new advanced sequences (3 D FIAS TA, FIASTA FATSAT), and these sequences did not extend the time of examination by more than $6 \mathrm{~min}$ and added significant value as they clarified the neural details from the nearby vessels and improved the visualization of the nerve along the entire pathways. We are hoping to add it to the MRN protocol for examining nerve pathways.

As a result of good agreement between the ED studies and the MRN, we recommended this MRN protocol be used in selective cases such as those with no detectable cause for brachial plexopathy. This could include postoperative patients or suspected tumor invasions that need further localization and determination of the nature of the lesion.

Our study had many limitations since the variations of the types of pathology causing brachial plexopathy result in a small sample size for each group affecting the degree of confidence of the statistical analysis. Histopathological evaluation and nerve biopsy are not common, and all cases should have an ED tests before the MRN examination as it would influence the MRN interpretation. More extensive prospective studies to establish the efficacy of the MRN and to explain the nature of these interesting radiological findings are warranted.

MRN significantly impacts the diagnostic and therapeutic management of patients with brachial plexopathy. It can consequently help the medical team select treatment (conservative or surgical), decide the most appropriate time to operate, and select the surgical technique. It also reduces operative time and thus allows for a better prognosis for these patients.
In most cases, MRN and ED results were agreed, and the combined anatomical and functional information obtained were complementary. MRN is a non-invasive imaging, and it represents a good alternative way for diagnosis in patients who cannot tolerate EMG. The actual utility of MRN will need to be determined.

\section{Conclusion}

MRN had an additive value to clinical examination and ED testes in the evaluation and localization of brachial plexus pathology, especially in cases of negative or inconclusive ED tests.

\section{Abbreviations}

MRN: Magnetic resonance neurography; ED: Electrophysiological studies; EMG: Electromyography; NCS: Nerve conduction study; PPV: Positive predictive value; NPV: Negative predictive value; TF: Indicates turbo factor; SPAIR: Spectral-attenuated inversion recovery; MPR: Multi-planar reconstruction; MIP: Maximum intensity projection

Acknowledgements

Staff member of Tanta University Hospital especially in the radiology.

\section{Authors' contributions}

RA correlated the study concept and design and had the major role in analysis. MS collected data in all stages of manuscript and performed data for analysis. ZH supervised the study with significant contribution to design of the methodology, manuscript revision, and preparation. FA correlated the finding with clinical finding and managed the cases. All authors have read and approved the final manuscript.

Funding

No funding.

Availability of data and materials

All data are available as sheets collected by authors.

\section{Declarations}

Ethics approval and consent to participate

Informed written consents taken from the patients, the study approved from ethical committee of faculty of medicine Tanta University number 1365-2018. 


\section{Consent for publication}

Not applicable.

\section{Competing interests}

The authors declare that they have no competing interests.

\section{Author details}

${ }^{1}$ Faculty of Medicine, Tanta University, Gharbia Governorate, Tanta, Algish Street, 31511, Egypt. ${ }^{2}$ Department of Radiology, Faculty of Medicine, Tanta University Hospital, Gharbia Governorate, Tanta, Algish Street, 31511, Egypt. ${ }^{3}$ Neurosurgery Department, Faculty of Medicine, Tanta University Hospital, Gharbia Governorate, Tanta, Algish Street, 31511, Egypt.

Received: 13 May 2021 Accepted: 6 July 2021

Published online: 26 July 2021

\section{References}

1. Vargas MI, Gariani J, Delattre BA, Dietemann JL, Lovblad K, Becker M (2015) Three-dimensional MR imaging of the brachial plexus. Semin Musculoskelet Radiol 19(2):137-148. https://doi.org/10.1055/s-0035-1546300

2. Hassan HG, Bassiouny RH, Mohammad SA (2018) Quantitative MR neurography of brachial plexus lesions based on diffusivity measurements. Egypt J Radiol Nuclear Med 49(4):1093-1102. https://doi.org/10.1016/j. ejrnm.2018.05.005

3. Aagaard BD, Maravilla KR, Kliot M (2001) Magnetic resonance neurography: magnetic resonance imaging of peripheral nerves. Neuroimaging Clin N Am 11:131-146

4. Van de Velde J, Bogaert S, Vandemaele P et al (2016) Brachial plexus 3D reconstruction from MRI with dissection validation: a baseline study for clinical applications. Surg Radiol Anat 38(2):229-236. https://doi.org/10.1007/ s00276-015-1549-x

5. Grant GA, Britz GW, Goodkin R, Jarvik JG, Maravilla K, Kliot M (2002) The utility of magnetic resonance imaging in evaluating peripheral nerve disorders. Muscle Nerve 25(3):314-331. 11870709. https://doi.org/10.1002/ mus. 10013

6. Yoshikawa T, Hayashi N, Yamamoto S, Tajiri Y, Yoshioka N, Masumoto T, Mori H, Abe O, Aoki S, Ohtomo K (2006) Brachial plexus injury: clinical manifestations, conventional imaging findings, and the latest imaging techniques. Radiographics 26(suppl-1):S133-S143. https://doi.org/10.1148/ rg.26si065511

7. Tagliafico A, Succio G, Serafini G et al (2012) Diagnostic accuracy of MRI in adults with suspect brachial plexus lesions: a multicenter retrospective study with surgical findings and clinical follow-up as reference standard. Eur J Radiol 81(10):2666-2672. https://doi.org/10.101 6/j.ejrad.2011.10.007

8. Bowen BC, Pattany PM, Saraf-Lavi E, Maravilla KR (2004) The brachial plexus: normal anatomy, pathology, and MR imaging. Neuroimaging Clin 14(1):5985. https://doi.org/10.1016/.nic.2003.12.002

9. Freund W, Brinkmann A, Wagner F, Dinse A, Aschoff AJ, Stuber G, Schmitz B (2007) MR neurography with multiplanar reconstruction of 3D MRI datasets: an anatomic study and clinical applications. Neuroradiology. 49(4):335-341. https://doi.org/10.1007/s00234-006-0197-6

10. Boden SD, McCowin PR, Davis DO et al (1990) Abnormal magnetic resonance scans of the cervical spine in asymptomatic subjects. J Bone Joint Surg Am 72:1178-1184 PMID: 2398088

11. Wiesel SW, Tsourmas N, Feffer H et al (1984) A Study of computer-assisted tomography. I. The incidence of CAT scans in an asymptomatic group of patients. Spine 9:549-551

12. Gad DM, Hussein MT, Omar NN et al (2020) Role of MRI in the diagnosis of adult traumatic and obstetric brachial plexus injury compared to intraoperative findings. Egypt J Radiol Nucl Med 51:195. https://doi.org/1 0.1186/s43055-020-00310-2

13. Fisher S, Wadhwa V, Manthuruthil C, Cheng J, Chhabra A (2016) Clinical impact of magnetic resonance neurography in patients with brachial plexus neuropathies. Br J Radiol 89(1067):20160503. https://doi.org/10.1259/bjr.201 60503

14. Smith AB, Gupta N, Strober J, Chin C (2008) Magnetic resonance neurography in children with birth-related brachial plexus injury. Pediatr Radiol 38(2):159-163. https://doi.org/10.1007/s00247-007-0665-0

15. Du R, Auguste Kl, Chin CT et al (2010) Magnetic resonance neurography for the evaluation of peripheral nerve, brachial plexus, and nerve root disorders. J Neurosurg 112(2):362-371. https://doi.org/10.31 71/2009.7.JNS09414

16. Yoshida T, Sueyoshi T, Suwazono S, Suehara M (2015) Three-tesla magnetic resonance neurography of the brachial plexus in cervical radiculopathy. Muscle Nerve 52(3):392-396. https://doi.org/10.1002/mus.24560

17. Upadhyaya V, Upadhyaya DN, Singh AK (2015) Magnetic resonance neurography of the brachial plexus. Indian J Plast Surg 48(2):129-137. https://doi.org/10.4103/0970-0358.163045

18. Somashekar D, Yang LJS, Ibrahim M, Parmar HA (2014) High-resolution MRI evaluation of neonatal brachial plexus palsy: a promising alternative to traditional CT myelography. AJNR Am J Neuroradiol 35(6):1209-1213. https://doi.org/10.3174/ajnr.A3820

\section{Publisher's Note}

Springer Nature remains neutral with regard to jurisdictional claims in published maps and institutional affiliations.

\section{Submit your manuscript to a SpringerOpen ${ }^{\circ}$ journal and benefit from:}

- Convenient online submission

- Rigorous peer review

- Open access: articles freely available online

- High visibility within the field

- Retaining the copyright to your article

Submit your next manuscript at $\boldsymbol{\nabla}$ springeropen.com 